\title{
La révolution 3D des cellules souches
}

\section{Fabrication in vitro d'épithéliums sensoriels de l'oreille}

Azel Zine ${ }^{1,2}$

> L'oreille interne abrite l'organe sensoriel de l'audition, la cochlée, et l'organe de l'équilibration, le vestibule (Figure 1). Les cellules sensorielles de l'oreille interne, ou cellules ciliées (CC), sont les cibles d'atteintes diverses incluant des atteintes ischémiques, toxiques, génétiques ou les pertes cellulaires liées à l'âge [1]. Au cours de la phylogenèse et pour des raisons encore non élucidées, les mammifères ont perdu la capacité de régénérer les cellules ciliées [2]. Ainsi, chez les mammifères y compris l'homme, le nombre de ces cellules est déjà prédéterminé avant la naissance, et leur perte ou atteinte, dans la cochlée comme dans le vestibule, entraînent un déficit sensoriel irréversible. Actuellement, le seul traitement d'un déficit profond de l'audition est prothétique et consiste en un implant cochléaire. Cet appareillage, qui rend certes de grands services pour pallier le handicap de la surdité, ne traite pas l'oreille atteinte, mais capte et module plutôt les signaux sonores avant de les transmettre à des électrodes qui stimulent directement les neurones auditifs résiduels. L'exploration de pistes thérapeutiques nouvelles visant le remplacement des cellules ciliées pourrait ouvrir des perspectives encourageantes de restauration cellulaire pour les fonctions auditive et vestibulaire $[3,4]$.

Les cellules souches embryonnaires pluripotentes (CSE) et les cellules pluripotentes induites (iPS) ont des propriétés uniques de prolifération et de différenciation. Elles constituent une source cellulaire prometteuse pour des thérapies variées (pathologie cardiaque, rétinopathies pigmentaires, maladies neurodégénératives, etc.) [5]. Dans une perspective d'application à l'oreille interne, des recherches ont été entreprises pour induire in vitro la différenciation des CSE et iPS en cellules ciliées [6]. Ces recherches utilisent des lignées de CSE et iPS qui expriment le gène rapporteur codant pour la GFP (green fluorescent protein), sous le contrôle de l'amplificateur du gène Atohl, gène dont l'expression est restreinte aux cellules ciliées dans l'oreille embryonnaire. Elles ont permis de définir les conditions in vitro de production de cellules ciliées fonctionnelles [6-8].

Très récemment, l'équipe du Pr Hashino (Université de l'Indiana, États-Unis) a franchi une étape supplémentaire importante en produisant un épithélium sensoriel de l'oreille interne à partir des CSE murines via une technologie de culture en 3D $[9,10]$. Les chercheurs ont réussi à produire un utricule, un épithélium sensoriel vestibulaire de l'oreille interne, contenant une couche apicale de cellules ciliées, une couche basale de cellules de soutien et des neurones qui n'avaient jusqu'à présent jamais été produits in vitro. Dans ce travail, les auteurs ont modifié un protocole de culture en 3D de corps embryoïdes, préalablement mis au point en reproduisant in vitro, à partir de CSE, la formation de dérivés complexes du neuroectoderme comme le cortex cérébral et la rétine [11]. Durant la neurulation, l'ectoderme définitif se subdivise en deux parties: le neuroectoderme, qui donne naissance au système nerveux central,
${ }^{1}$ UMR 7260 CNRS, neurosciences intégratives et adaptatives, Aix-Marseille Université, Marseille, France ;

2 Faculté de Pharmacie, Université de Montpellier, Montpellier, France. azel.zine@inserm.fr

et l'ectoderme non-neural, qui génère diverses placodes neurogéniques ( $p l a-$ code olfactive, cristallinienne, etc.), dont la placode otique, à l'origine de l'oreille interne (Figure 2A). Grâce à une lecture très fine du développement embryonnaire de l'oreille interne de la souris in vivo, les auteurs ont pu en reproduire les étapes clés dans le système de culture en $3 \mathrm{D}$.

D'un point de vue méthodologique (Figure 2B), les auteurs ont d'abord déclenché une brève induction ectodermique en cultivant des CSE dans un milieu qui favorise la différenciation de l'ectoderme en présence de matrigel, gel contenant des protéines de la matrice extracellulaire. Après deux jours en présence de matrigel, les agrégats cellulaires formés ont constitué un épithélium de type ectoderme plus ou moins arrondi au niveau de la partie externe. À partir du $3^{\mathrm{e}}$ jour in vitro, et en réponse à la modulation de deux voies de signalisation intervenant dans le développement (inhibition de la voie TGF $\beta$ [transforming growth factor $\beta$ ] et stimulation concomitante de la voie BMP [bone morphogenetic protein]), les agrégats de cellules exprimaient des caractéristiques d'ectoderme non-neural. Durant l'embryogenèse, l'activation de la voie des BMP est cruciale pour I'induction de l'ectoderme non-neural à partir de l'ectoderme définitif [12]. En accord avec ce rôle de la voie BMP au cours du développement in vivo, on observe, à partir du $3^{e}$ jour in vitro dans les cellules des agrégats traités par BMP4, une surexpression de DIx3 


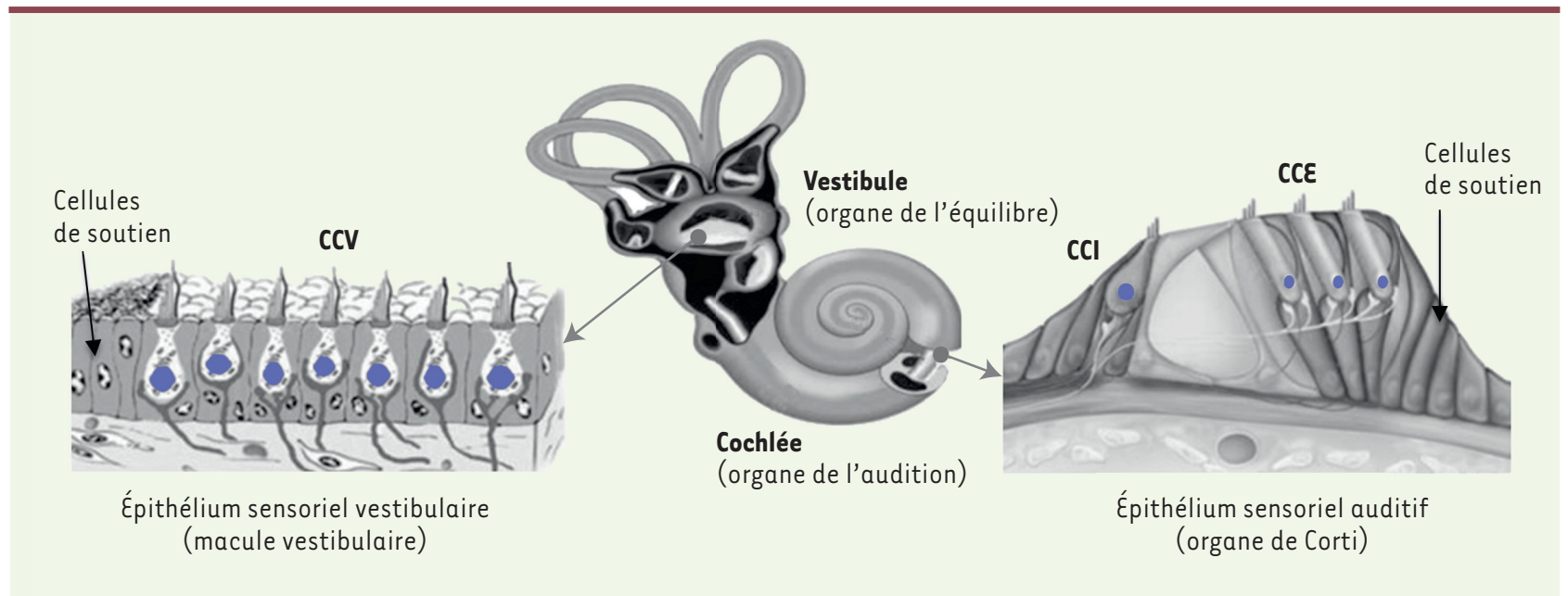

Figure 1. Représentation schématique de l'oreille interne (vestibule et cochlée). La cochlée capte les sons et le vestibule détecte les accélérations par des mécanorécepteurs, les cellules ciliées. Dans l'épithélium sensoriel auditif (organe de Corti), les cellules ciliées internes (CCI) sont disposées en une seule rangée et les cellules ciliées externes (CCE) en trois rangées. Dans l'épithélium sensoriel vestibulaire, les cellules ciliées vestibulaires (CCV) tapissent la surface de la macule. Des cellules de soutien des épithéliums sensoriels de l'oreille interne entourent les cellules sensorielles.

(marqueur de l'ectoderme non-neural), et une diminution de Soxl (marqueur du neuroectoderme). Puis, en traitant les cellules avec des inhibiteurs de la voie BMP et des molécules FGF (fibroblast growth factor), les auteurs sont parvenus à différencier l'ectoderme nonneural vers un épithélium préplacodal, évoluant ensuite vers une placode/vésicule otique. Après ces deux phases d'induction successives, les agrégats ont été transférés dans des conditions de cultures flottantes dans un milieu basal sans sérum. Au cours de cette dernière phase de différenciation, certaines cellules prosensorielles exprimant des marqueurs de la vésicule otique in vivo (dont Pax2 et Pax8) se sont différenciées en cellules ciliées, en cellules de soutien et en neurones innervant les cellules ciliées. En résumé, tous les types cellulaires composant un épithélium sensoriel de l'oreille interne ont été produits dans ce modèle de culture 3D.

Durant le développement in vivo, l'invagination de la placode otique donne naissance à la forme la plus précoce de l'oreille interne, la vésicule otique, qui se divise ensuite en deux composantes, ventrale et dorsale. La composante ven- trale donne naissance à la cochlée et la dorsale au système vestibulaire. Or, in vitro, les cellules ciliées différenciées après la dernière étape exprimaient des propriétés morphologiques et des marqueurs caractéristiques de cellules vestibulaires (Figure 1). Les auteurs ont également observé que les neurones marqués avec des anticorps reconnaissant la tubuline $\beta$ III et les neurofilaments se développaient dans des endroits distincts le long de l'épithélium vestibulaire. L'analyse du pôle basal des cellules ciliées a confirmé la présence de synapses associées à une protéine CtBP2 ( $C$-terminal binding protein 2), un marqueur des synapses à ruban des cellules sensorielles de l'oreille. Outre ces propriétés morphologiques, ces cellules ciliées expriment la plupart des propriétés physiologiques de leurs homologues in vivo. Les auteurs concluaient que leur méthode de culture 3D était plus efficace que d'autres modèles de différenciation in vitro, car elle produisait un nombre supérieur de cellules ciliées, en moyenne 1500 par agrégat. Considérant que chaque oreille interne contient 30000 cellules ciliées dans l'ensemble des deux composantes cochléaire et vestibulaire, 20 agrégats issus du protocole d'induction en culture 3D pourraient fournir un nombre de cellules ciliées correspondant à la totalité des cellules ciliées de l'oreille interne. Cependant, la différenciation préférentielle des CSE vers un phénotype de type vestibulaire indique que d'autres facteurs d'induction otique sont nécessaires pour parvenir à générer un épithélium sensoriel de type auditif in vitro.

Il est prématuré de prédire la place qu'occupera la thérapie cellulaire parmi les approches thérapeutiques en cours de développement pour restaurer l'audition chez les patients atteints d'une surdité liée à la perte de cellules sensorielles. Néanmoins, ce nouveau modèle de culture $3 D$ permet de reproduire à l'identique l'organogenèse de l'oreille interne, et pourrait s'avérer utile pour enrichir notre compréhension du développement normal et pathologique de l'oreille interne. II pourrait également servir à cribler des molécules d'intérêt dans le domaine de l'équilibre et l'audition. $\diamond$

3D revolution of stem cells: in vitro generation of ear sensory epithelia

\section{LIENS D'INTÉRÊT}

Les auteurs déclarent n'avoir aucun lien d'intérêt concernant les données publiées dans cet article. 
A

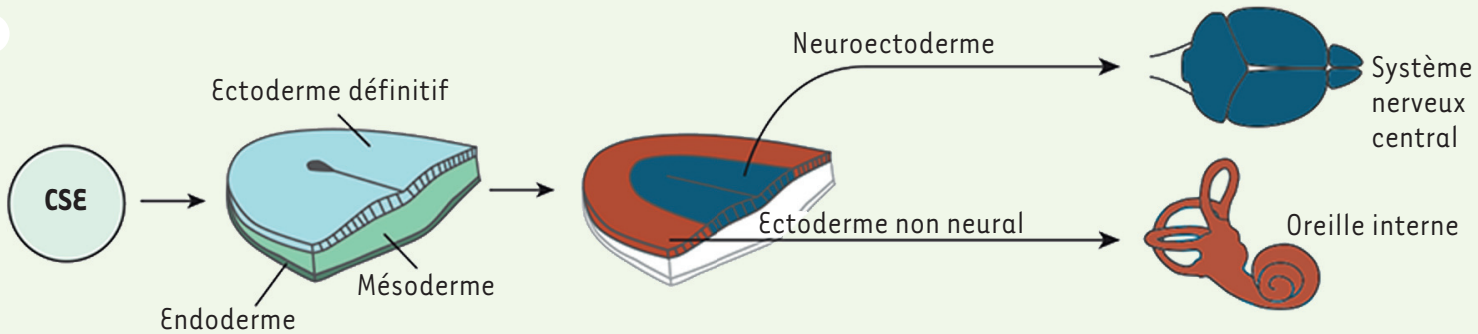

B

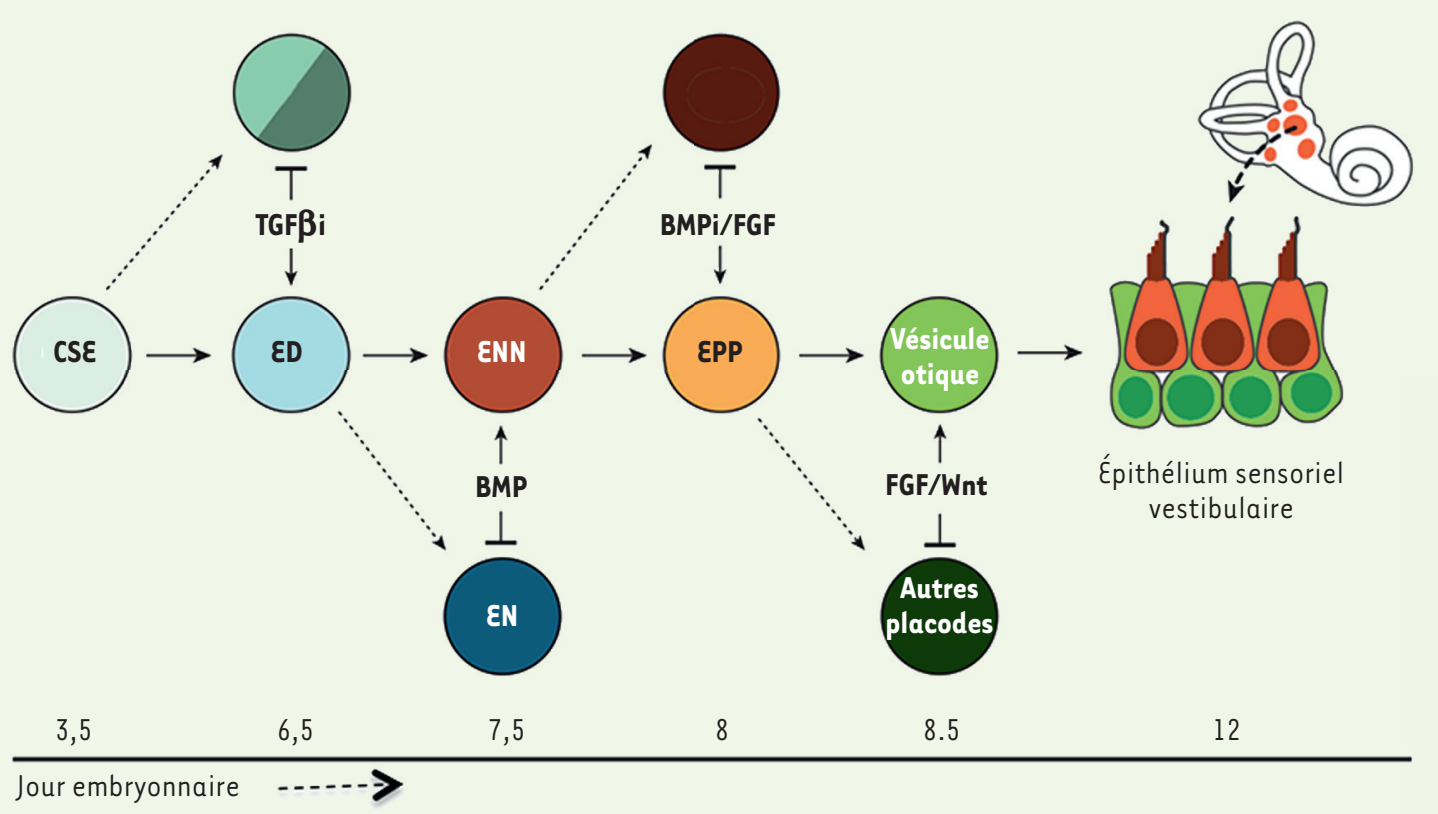

Figure 2. Développement de l'oreille interne à partir de l'ectoderme définitif. A. Les interactions entre l'ectoderme non-neural et le neuroectoderme interviennent très tôt au cours du développement embryonnaire. Ces interactions sont à l'origine de la ségrégation des lignages de cellules qui vont adopter des destins différents : système nerveux central (SNC) et oreille interne. B. Les voies de signalisation TGF $\beta$, FGF et Wnt sont parmi les voies majeures impliquées dans l'induction otique au cours du développement in vivo et in vitro. Le terme «otique 》 désigne spécifiquement les cellules prosensorielles présentes dans la placode/vésicule otique à l'origine des épithéliums sensoriels de l'oreille interne. « Autres placodes » se réfèrent aux placodes adénohypophysaire, cristallinienne, olfactive, trigéminale et épibranchiales. CSE : cellule souche embryonnaire; $\varepsilon D$ : ectoderme définitif ; ENN : ectoderme non-neural ; $\varepsilon P P$ : épithélium pré-placodal ; TGF $\beta$ : transforming growth factor $\beta$; FGF : fibroblast growth factor; BMP : bone morphogenetic protein ; BMPi : inhibition de la voie BMP ; TGF $\beta$ i : inhibition de la voie TGF $\beta$ (adapté avec la permission de Nature Publishing Group [9]).

\section{REMERCIEMENTS}

L'auteur remercie le Dr Isabelle Virard (UMR 7259, Aix-Marseille Université) pour sa relecture et ses commentaires et s'excuse après des auteurs qui n'ont pas pu être cités, faute de place.

\section{RÉFÉRENCES}

1. Legendre K, Petit C, દl-Amraoui A. La cellule ciliée externe de la cochlée des mammifères. Med Sci (Paris) $2009 ; 25: 117-20$.

2. Fritsch B, Beisel KW, Hansen LA. The molecular basis of neurosensory cell formation in ear development: a blueprint for hair cell and sensory neuron regeneration? Bioessays $2006 ; 28$ : 1181-93.
3. Zine A, Lowenheim H, Fritzsch B. Toward translating molecular ear development to generate hair cells from Stem Cells. In : Kursad T, ed. Adult stem cells. New York : Springer Science, 2014 : 111-61.

4. Géléoc GS, Holt JR. Sound strategies for hearing restoration. Science $2014 ; 344: 1241062$.

5. Wichterle H, Przedborski S. What can pluripotent stem cells teach us about neurodegenerative diseases? Nat Neurosci $2010 ; 13: 800-4$.

6. Oshima K, Shin K, Diensthuber M, et al. Mechanosensitive hair cell-like cells from embryonic and induced pluripotent stem cells. Cell $2010 ; 141: 704-16$.

7. Zine A. Des cellules souches pour corriger les surdités neurosensorielles? Med Sci (Paris) $2004 ; 20: 518-20$.

8. El-Amraoui A, Petit C. Thérapie cellulaire dans l'oreille interne : nouveaux développements et perspectives. Med Sci (Paris) $2010 ; 26: 981-5$.
9. Koehler KR, Mikosz AM, Molosh Al, et al. Generation of inner ear sensory epithelia from pluripotent stem cells in 3D culture. Nature $2013 ; 500: 217-21$.

10. Koehler KR, Hashino $\varepsilon$. 3D mouse embryonic stem cell culture for generating inner ear organoids. Nat Protoc $2014 ; 9$ : 1229-44.

11. Eiraku M, Sasai Y. Mouse embryonic stem cell culture for generation of three-dimensional retinal and cortical tissues. Nat Protoc $2011 ; 7$ : 69-79.

12. Kwon, HJ, Bhat, N, Sweet EM, et al. Identification of early requirements for preplacodal ectoderm and sensory organ development. PLoS Genet $2010 ; 6$ : el001133. 